\title{
A Study on the Mechanism of Impact between Curved Bridge Segments Using Nonsmooth Dynamics
}

\author{
Wenshan Li $(\mathbb{D}$, Yong Huang $\mathbb{D}$, and Guangming Xie \\ Institute of Engineering Mechanics, China Earthquake Administration, Harbin 150000, China \\ Correspondence should be addressed to Yong Huang; huangyong@iem.ac.cn
}

Received 9 February 2020; Revised 29 November 2020; Accepted 6 December 2020; Published 17 December 2020

Academic Editor: Marco Lepidi

Copyright ( $\odot 2020$ Wenshan Li et al. This is an open access article distributed under the Creative Commons Attribution License, which permits unrestricted use, distribution, and reproduction in any medium, provided the original work is properly cited.

\begin{abstract}
It has been observed in many previous earthquakes that impact often occurs between the main girders in curved bridges. An earthquake can result in deck-unseating leading to catastrophic destruction of the structure. In this paper, the nonsmooth multirigid body dynamics method and the set-valued formulation were used to model and analyze the mechanism of impact between the curved bridge segments. The analysis demonstrated that these impacts are the major cause of segment rotation. The main contribution of this paper is to use Newton's impact law and Coulomb's friction law to describe the interaction between the curved bridge segments in the form of a setvalued function and to express impacts with friction as a linear complementary problem. For frictionless and frictional contact, the paper considers the single-point and multipoint impacts using the linear complementary formula to detect the unique actual slip-stick conditions of these states. A variety of criteria for distinguishing each case are presented and the results provide the kinetic characteristics of each contact case. The analysis has shown that the impact between the segments of a curved bridge and the tendency of the segments to rotate (and thus detach) are related to the overall geometry, the coefficient of restitution, the coefficient of friction, and the preimpact conditions in the plane of motion. Finally, a theoretical relationship diagram of the impact, rotation slip, and stick condition of the curved bridge segments at the contact point is given. The presented results will be useful for the seismic design of curved bridges.
\end{abstract}

\section{Introduction}

Impact occurs when two or more bodies contact for a brief time, which is mainly determined by the material characteristics, geometry, and preimpact conditions of the bodies. Pounding between the adjacent segments, in-plane deck rotation, and deck-abutment interaction make the curved bridges very susceptible during such activities, resulting in deck-unseating, shear failure, torsional failure, and interfacial concrete failure [1-4].

Impacts between curved bridge segments during strong earthquakes have been reported for many structures, for example, the I-5/SR-14 interchange bridge during the 1971 San Fernando earthquake, the I-10/I-215 interchange bridge during the 1991 San Francisco earthquake in the United States [5], Southbound Separation Overpass during the United States Northridge earthquake [6], Kobe Line and Shibukawa Interchange Bridge No. 3 during the 1995 Kobe earthquake in Japan [7], and the Shiwei Bridge and Maoluoxi
Bridge during the 1999 Taiwan Chi-Chi earthquake. The Baihua Bridge [8, 9], shown in Figure 1, sustained torsional damage of the pier and deck-unseating during the 2008 Wenchuan earthquake in China. The same 2008 Wenchuan earthquake damaged curved ramp bridges of the Huilan interchange [10].

The reviewed earthquake damage cases demonstrate that it is necessary to investigate the impact between the curved bridge superstructures during strong ground motion events, and a significant number of relevant studies have already been conducted. Using the Karnopp friction model, Amjadian and Agrawal [11] investigated the influence of pounding on rigid-body motion of horizontally curved bridges during strong earthquakes. Banerjee et al. [12] studied the dynamic response sensitivity of horizontally curved bridges under seismic impact and assessed the influence of columns, bent arrangement, and gap distances on the structural response. Julian et al. [13] studied the rotations and displacements of curved bridge superstructures caused 


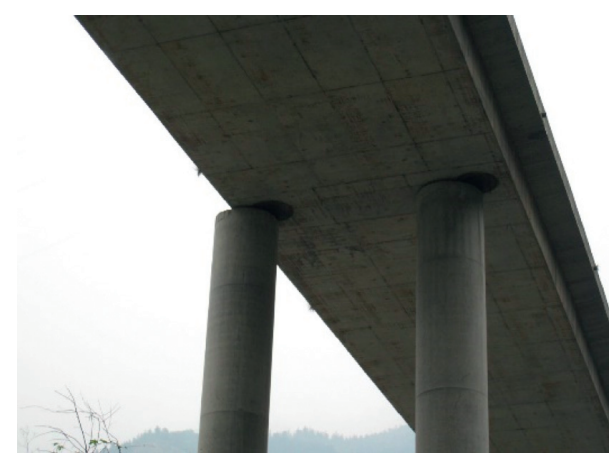

Figure 1: Unseating of Baihua Bridge caused the Wenchuan earthquake.

by flexural-torsional interaction under seismic loading. A two-fifths scale curved bridge model was constructed at the University of Nevada, Reno Large Scale Structures Laboratory, and tested on a shake table to observe the effects of earthquake shocks and develop a contact element for design [14].

There are many impact simulation methods $[4,15]$, such as the restitution coefficient method, the stereo-mechanical approach, the compliance method, and the linear complementarity problem (LCP) method. The restitution coefficient method [16] uses the principle of conservation of momentum to modify the velocity after impact according to the precontact state of the colliding bodies. The restitution coefficient method has the advantages of conceptually clarity and calculation simplicity. However, the method only uses the coefficient of restitution to model the changes in structural strength and speed before and after the impact and not during the whole impact process. Thus, the method can simulate the impact process of rigid bodies, but not deformable objects. Although the stereo-mechanical approach has the advantages of conceptual clarity and simple numerical implementation, it cannot consider the compression deformations of the contact areas. The stereo-mechanical approach can be applied when the stiffness of the impacting objects is very large and the impact time is very short. However, when the stiffness of the objects is very small, they will be more prone to large deformations and the impact between them will last longer. In the compliance method, contact elements are used to simulate the contact impact. The commonly used contact elements are Kelvin elements $[17,18]$ and Hertz elements $[19,20]$. The advantage of the compliance method is the convenient calculation and clear physical meaning and that the approach can be used in combination with finite element software. However, in the process of a simulating impact, the model will be affected by the material and structural shape, and the choice of these parameters is left to the subjective judgment of the analyst. If the contact stiffness is large, convergence becomes relatively quick, but the calculated impact force will be unrealistic. On the other hand, if the contact stiffness is small, the impact time will be longer, and the calculated impact force is relatively small. Because of its uncertainty, the damping coefficient cannot accurately simulate the energy loss of adjacent structures during impact. In 1988, Moreau, known as the father of nonsmooth mechanics, published his seminal paper [21]. Panagiotopoulos [22, 23] improved this new theory by introducing a nonconvex variational inequality. After the Delassus problem [24] was posed in 1917, researchers realized that as the number of contact points increases, and it is impossible to make a real state in a large number of possible contact states. However, the LCP method can detect the hysteresis and contact states in the nonsmooth events unambiguously and effectively; thus, it is convenient to use the LCP method to study nonsmooth dynamics phenomena. Pfeifer and Glocker [25] successfully combined multibody system dynamics with nonsmooth mechanics theory, which laid the theoretical foundation for nonsmooth multibody system dynamics. Dimitrakopoulos $[26,27]$ proposed a method for analyzing abutment-to-deck and deck-to-deck impact in skewed bridges. This paper studies in detail the impact on curved bridges based on their method.

In this formalism, the deck is assumed to be a rigid body. Therefore, any impacts that may occur between the decks are locally convex. The problem of impacts in the curved bridge in the LCP method is transformed by a complementary form of the normal Newton impact law [25], the tangential Coulomb friction law, and the set-valued function. By solving the LCP equation, the normal and transverse stickslip contact cases of curved bridge segments are studied for different preimpact conditions. The overall geometry influences significantly the postimpact deck responses, which again depended on the preimpact conditions. This complex phenomenon is analytically studied for a single-impact case during deck-to-deck pounding between two curved bridge segments. Based on a previous investigation [12] on the impact between the abutment and the deck of a curved bridge, this paper studies the problem of impact between bridge segments of a curved bridge. The main contributions are as follows: first, the mechanism of deck rotation caused by the impact between bridge segments of a curved bridge is explained. Next, the specific conditions to be satisfied to avoid the rotation of the deck when friction is ignored are given. Finally, the classification boundary of slip and stick after two adjacent bridge segments make contact is established from a kinematic consideration.

\section{Research Methods}

2.1. Linear Complementarity Problem. The LCP method can encapsulate many of the physical phenomena occurring during bridge segment impacts in a curved bridge. In this paper, by solving the LCP equation, the deck of the bridge is assumed to be a fully rigid body moving only in the horizontal plane [25]. This study further assumes that the interaction between adjacent parts is a unilateral contact [25]. This interaction is described in the form of the set-valued function using Newton's impact law in the normal direction and Coulomb's friction law in the transverse direction. Only two impact parameters are required to describe the contact, namely, the coefficient of restitution, $\varepsilon_{N}$, and the coefficient of friction, $\mu$. The coefficient of restitution, $\varepsilon_{N}$, is defined as the ratio of postimpact normal relative velocity $\dot{g}_{N}^{+}$, to 
preimpact normal relative velocity, $\dot{g}_{N}^{-}$. It can vary between 0 and 1; that is, $\varepsilon_{N} \in[0,1]$. The case $\varepsilon_{N}=0$ corresponds to the completely inelastic impact and $\varepsilon_{N}=1$ to the fully elastic impact. In the transverse direction, a zero coefficient of restitution is assumed, $\varepsilon_{T}=0$. Newton's impact law can be represented as $\dot{g}_{N}^{+}=-\varepsilon_{N} \dot{g}_{N}^{-}[25]$.

The set-valued function is an extension of the ordinary function concept. The set-valued function $F: R^{n} \longrightarrow R^{n}$ is a multivalued mapping between the domain of definition and the range, which maps point $x \in \mathbf{R}^{n}$ in the domain of definition to the aggregate $F(x) \in \mathbf{R}^{n}$ in the value domain [25].

The normal impulse, $\Lambda_{N i}$, and the transverse impulse, $\Lambda_{T i}$, use two set-valued mappings, the unilateral primitive function, Upr (Figure 2, right) and the sgn function (Figure 2, left), respectively:

$$
-\Lambda_{N i} \in \operatorname{Upr}\left(v_{N i}\right)-\Lambda_{T_{i}} \in \mu_{i} \Lambda_{N i} \operatorname{sgn}\left(v_{T i}\right) .
$$

The sgn function is an extension of the normal signum function. The difference is that the function maps the parameter $x=0$ to the set $[-1,+1]$. The image of the sgn function at $x=0$ is a vertical line segment. The sgn function can be decomposed into two unilateral primitive functions [25]. The following decomposition is also illustrated in Figure 2:

$$
\begin{cases}-\Lambda_{T R i} \in U \operatorname{Upr}\left(v_{T R i}\right), & \Lambda_{T R i}=\mu \Lambda_{N i}+\Lambda_{T i} \\ -\Lambda_{T L i} \in U \operatorname{Pp}\left(v_{T L i}\right), & \Lambda_{T L i}=\mu \Lambda_{N i}-\Lambda_{T i}, \\ \dot{g}_{T i}^{+}=v_{T R i}-v_{T L i} . & \end{cases}
$$

Expressing the above system of equations is a matrix form yielding

$$
\boldsymbol{\Lambda}_{T L}=\overline{\bar{\mu}} \boldsymbol{\Lambda}_{N}-\boldsymbol{\Lambda}_{T}=2 \overline{\bar{\mu}} \Lambda_{N}-\Lambda_{T R},
$$

where $\overline{\bar{\mu}}=\operatorname{diag}\left\{\mu_{i}\right\}$. The LCP method can parametrize the system using a linear system of equations, $\mathbf{y}=\mathbf{A x}+\mathbf{b}$, with a known matrix $\mathbf{A}$ and vector $\mathbf{b}$. Vectors $\mathbf{y}$ and $\mathbf{x}$ are unknown. The additional complementarity is defined by $\mathbf{y} \geq 0, \mathbf{x} \geq 0$, and $\mathbf{y}^{\mathrm{T}} \mathbf{x}=0$. More detailed information about the LCP, the solvability of the numerical LCP, and the associated numerical algorithms are available in $[28,29]$.

Using Newton's impact law and Coulomb's friction law, a coupled set of linear complementary equations can be expressed as follows [30]:

$$
\begin{aligned}
& \left(\begin{array}{c}
\mathbf{v}_{N} \\
\mathbf{v}_{T R} \\
\boldsymbol{\Lambda}_{T L}
\end{array}\right)=\left(\begin{array}{ccc}
\mathbf{G}_{N N}-\mathbf{G}_{N T} \overline{\bar{\mu}} & \mathbf{G}_{N T} & 0 \\
\mathbf{G}_{T N}-\mathbf{G}_{T T} \overline{\bar{\mu}} & \mathbf{G}_{T T} & \mathbf{E} \\
2 \overline{\bar{\mu}} & -\mathbf{E} & 0
\end{array}\right)\left(\begin{array}{c}
\boldsymbol{\Lambda}_{N} \\
\boldsymbol{\Lambda}_{T R} \\
\mathbf{v}_{T L}
\end{array}\right) \\
& +\left(\begin{array}{c}
\left(\overline{\bar{\varepsilon}}_{\mathbf{N}}+\mathbf{E}\right) \dot{g}_{T}^{-} \\
\mathbf{E} \cdot \dot{g}_{T}^{-} \\
0
\end{array}\right) \\
& \left(\begin{array}{c}
\mathbf{v}_{N} \\
\mathbf{v}_{T R} \\
\boldsymbol{\Lambda}_{T L}
\end{array}\right) \geq 0 \\
& \left(\begin{array}{c}
\boldsymbol{\Lambda}_{N} \\
\boldsymbol{\Lambda}_{T R} \\
\mathbf{v}_{T L}
\end{array}\right) \geq 0, \\
& \left(\begin{array}{c}
\mathbf{v}_{N} \\
\mathbf{v}_{T R} \\
\boldsymbol{\Lambda}_{T L}
\end{array}\right)^{T}\left(\begin{array}{c}
\boldsymbol{\Lambda}_{N} \\
\boldsymbol{\Lambda}_{T R} \\
\mathbf{v}_{T L}
\end{array}\right)=0
\end{aligned}
$$

where $\overline{\bar{\varepsilon}}_{\mathbf{N}}=\operatorname{diag}\left\{\varepsilon_{N i}\right\}, \overline{\bar{\mu}}=(\mu \mu)$, and $\mathbf{E}$ is an identity matrix.

2.2. Geometric Analysis. In the kinematic analysis of bridge impact, we can represent the possible contact point relative distance, $g_{i}$, and the relative speed, $\dot{g}_{t}$, as generalized coordinates $\mathbf{q}^{i}$. Since a rigid body on a plane has three degrees of freedom, the generalized coordinate vector can be expressed as $\mathbf{q}^{T}=\left[\begin{array}{llllll}x_{1} & y_{1} & \theta_{1} & x_{2} & y_{2} & \theta_{2}\end{array}\right]$. Figure 3 illustrates the position of the left deck (deck-1) and the right deck (deck-2).

The distance from the center of mass to the center of the bridge curvature is

$$
d_{m i}=\left(2+\frac{k^{2}}{6}\right) \frac{\sin \left(\alpha_{i} / 2\right)}{\alpha_{i}} R, \quad i=1,2,
$$

where $k=(W / R)$ and $i=1,2$ indicate deck-1 and deck-2, respectively. From this formula, the moment of inertia can be calculated as follows:

$$
I_{i}=\left(1+\frac{1}{4} k^{2}\right) L_{i} R^{2}, \quad i=1,2,
$$

where $L_{i}=k \alpha_{i} R^{2}$. Figure 4 shows that the distance vector, $\mathbf{r}_{\mathrm{D}}$, between deck-1 and deck-2 is determined by the parameters $\sigma_{1}$ and $\sigma_{2}$ :

$$
\mathbf{r}_{\mathrm{D}}=\left(\begin{array}{c}
\delta+x_{2}-x_{1}+R\left(\sin \theta_{1}-\sin \theta_{2}\right)+\sigma_{1} \cdot \sin \theta_{1}-\sigma_{2} \cdot \sin \theta_{2} \\
+d_{m 2} \cdot \sin \left(\theta_{2}-\frac{\alpha_{2}}{2}\right)+d_{m 2} \cdot \sin \frac{\alpha_{2}}{2}-d_{m 1} \cdot\left[\sin \frac{\alpha_{1}}{2}+\cos \left(\theta_{1}-\frac{\alpha_{1}}{2}\right)\right] \\
y_{2}-y_{1}+R\left(\cos \theta_{2}-\cos \theta_{1}\right)+\sigma_{2} \cdot \cos \theta_{2}-\sigma_{1} \cdot \cos \theta_{1} \\
-d_{m 2} \cdot \cos \left(\theta_{2}-\frac{\alpha_{2}}{2}\right)+d_{m 2} \cdot \cos \frac{\alpha_{2}}{2}-d_{m 1} \cdot\left[\cos \frac{\alpha_{1}}{2}-\cos \left(\theta_{1}-\frac{\alpha_{1}}{2}\right)\right]
\end{array}\right) .
$$




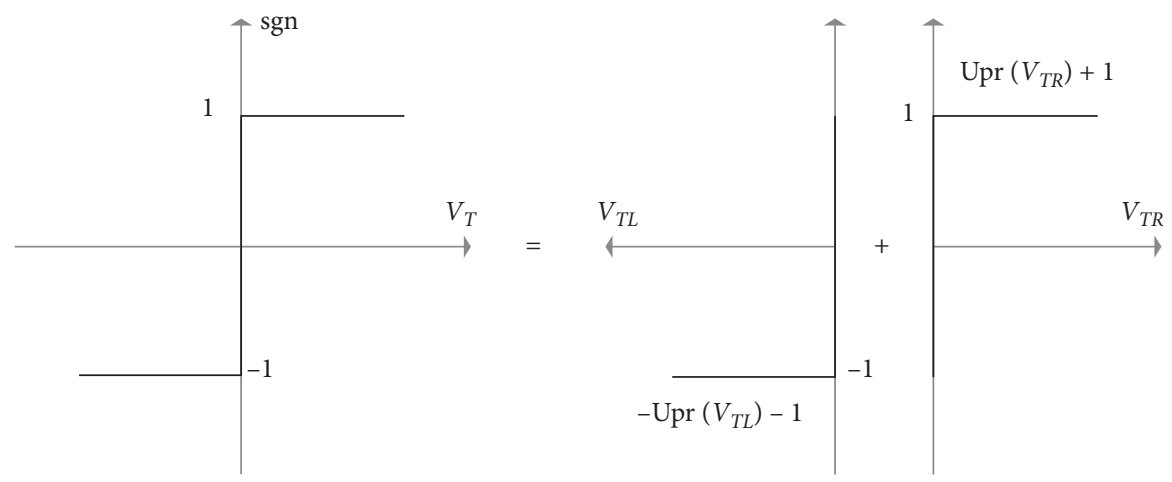

FIgURE 2: Set-valued friction force law.

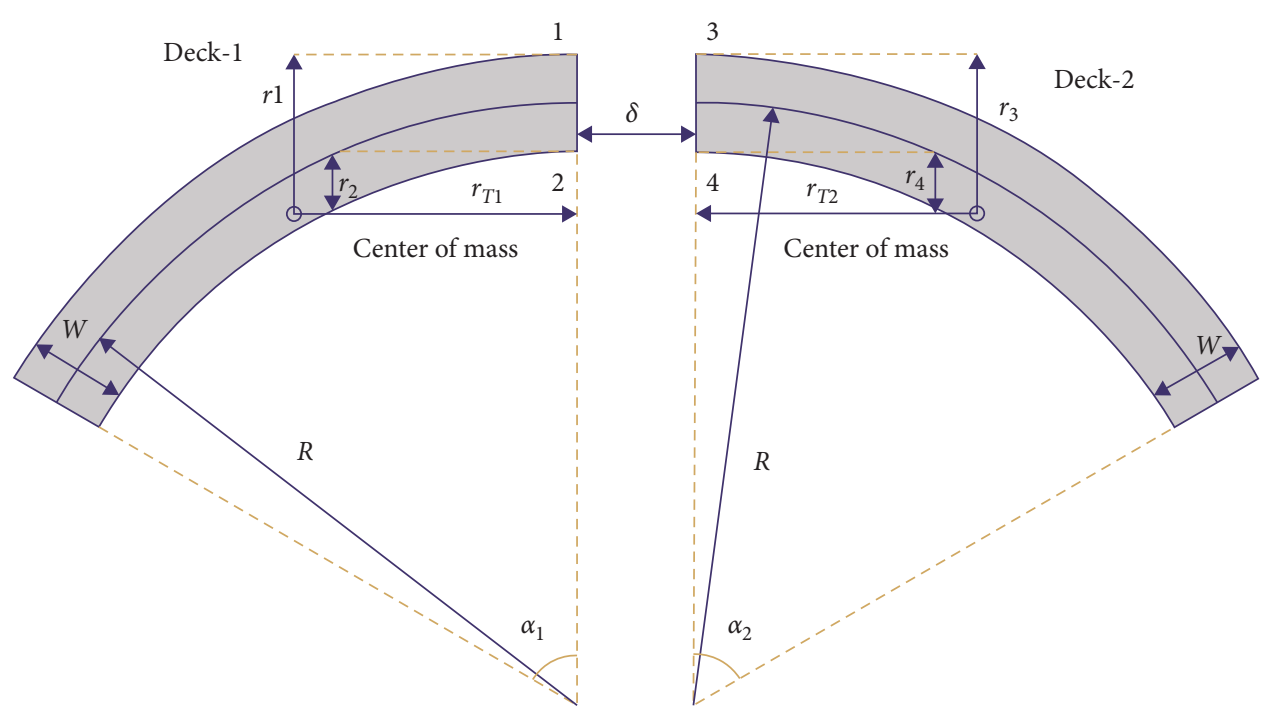

FIGURE 3: Deck geometry of curved bridge showing the position of the center of mass and different impact parameters without in-plane deck rotation for deck-1 and deck-2.

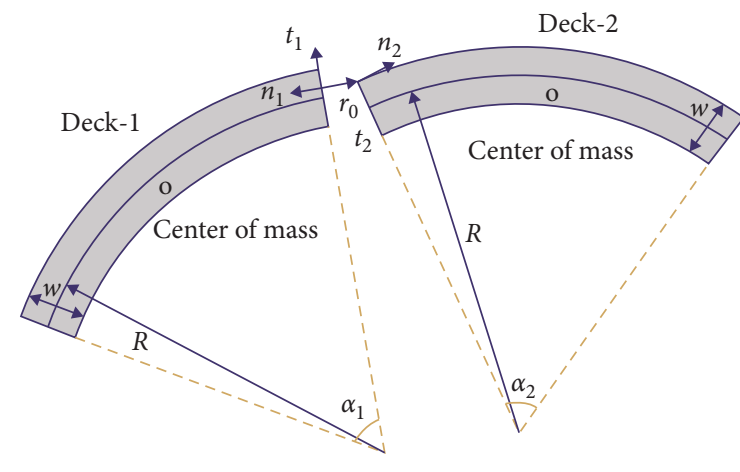

(a)

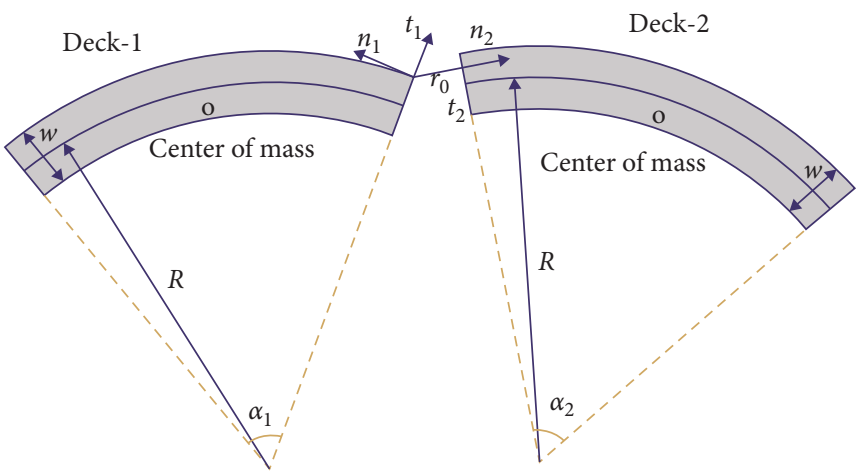

(b)

Figure 4: Contact between deck-1 and deck-2. (a) Case 1. (b) Case 2. 
In this formula, $\sigma_{i}$ describes the location of the contact point at the contour of the deck. The range of $\sigma_{i}$ is between $-0.5 \mathrm{~W}$ and $+0.5 \mathrm{~W}$. The tangential and normal vectors of the contour of deck- 1 and deck- 2 can now be calculated as follows:

$$
\begin{aligned}
\mathbf{t}_{1} & =\left(\begin{array}{c}
-\sin \theta_{1} \\
\cos \theta_{1}
\end{array}\right), \\
\mathbf{t}_{2} & =\left(\begin{array}{c}
\sin \theta_{2} \\
-\cos \theta_{2}
\end{array}\right), \\
\mathbf{n}_{1} & =\left(\begin{array}{c}
-\cos \theta_{1} \\
-\sin \theta_{1}
\end{array}\right), \\
\mathbf{n}_{2} & =\left(\begin{array}{c}
\cos \theta_{2} \\
\sin \theta_{2}
\end{array}\right),
\end{aligned}
$$

where $\mathbf{t}_{1}, \mathbf{t}_{2}, \mathbf{n}_{1}$, and $\mathbf{n}_{2}$ represent the tangential and normal direction vectors of the two contours, as shown in Figure 4.

\section{Kinematic Analysis}

This section discusses the kinematic problem between two adjacent bridge segments of a curved bridge. In the following analysis, the contact between deck-1 and deck-2 is divided into multiple-point contact (the impact takes place along a contour) and single-point contact (the impact takes place in a corner). We can then analyze the single-point contact in two specific cases.

Case 1. A point on deck-2 touches the contour of deck-1.

When this happens, $\mathbf{r}_{D}^{T} \mathbf{t}_{1}=0$ is satisfied, as shown in Figure 4(a). In this case, we can calculate the normal relative displacement, $g_{N}$, as follows:

$$
\begin{aligned}
g_{N}= & -\mathbf{r}_{D}^{T} \mathbf{n}_{1}=\left(\delta+x_{2}-x_{1}\right) \cdot \cos \theta_{1}+\left(y_{2}-y_{1}\right) \\
& \cdot \sin \theta_{1}+\left(R+\sigma_{2}\right) \cdot \sin \left(\theta_{1}-\theta_{2}\right) \\
& +d_{m 2} \cdot\left[\sin \left(\theta_{1}+\frac{\alpha_{2}}{2}\right)+\sin \left(\theta_{2}-\theta_{1}-\frac{\alpha_{2}}{2}\right)\right] \\
& -d_{m 1} \cdot\left[\sin \left(\theta_{1}+\frac{\alpha_{1}}{2}\right)-\sin \frac{\alpha_{1}}{2}\right] .
\end{aligned}
$$

Using $\dot{g}_{N}=\mathbf{W}_{N}^{T} \mathbf{q}$, calculate the normal constrained Jacobian matrix, $\mathbf{W}_{N}^{T}$, as follows:

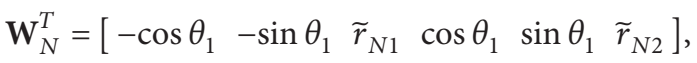

$$
\begin{aligned}
& \widetilde{r}_{N 1}=\left(x_{1}-x_{2}-\delta\right) \sin \theta_{1}+\left(y_{2}-y_{1}\right) \cos \theta_{1} \\
& +\left(R+\sigma_{2}\right) \cos \left(\theta_{1}-\theta_{2}\right)+d_{m 2} \text {, } \\
& \cdot\left[\cos \left(\theta_{1}+\frac{a_{2}}{2}\right)-\cos \left(\theta_{2}-\theta_{1}-\frac{a_{2}}{2}\right)\right] \\
& -d_{m 1} \cdot \cos \left(\theta_{1}+\frac{a_{1}}{2}\right) \text {, } \\
& \widetilde{r}_{N 2}=-\left(R+\sigma_{2}\right) \cos \left(\theta_{1}-\theta_{2}\right)+d_{m 2} \cdot \cos \left(\theta_{2}-\theta_{1}-\frac{\alpha_{2}}{2}\right) \text {. }
\end{aligned}
$$

Case 2. A point on deck-1 touches the contour of deck-2.

This happens when $\mathbf{r}_{D}^{T} \mathbf{t}_{2}=0$ is satisfied and is illustrated in Figure 4(b). One can calculate the normal relative displacement, $g_{N}$, as follows:

$$
\begin{aligned}
g_{N}= & r_{D}^{T} n_{2}=\left(\delta+x_{2}-x_{1}\right) \cdot \cos \theta_{2}+\left(y_{2}-y_{1}\right) \cdot \sin \theta_{2} \\
& +\left(R+\sigma_{1}\right) \cdot \sin \left(\theta_{1}-\theta_{2}\right) \\
& +d_{m 2} \cdot\left[\sin \left(\theta_{2}+\frac{\alpha_{2}}{2}\right)-\sin \left(\frac{\alpha_{2}}{2}\right)-d_{m 1}\right. \\
& \cdot\left[\sin \left(\theta_{2}+\frac{\alpha_{1}}{2}\right)-\sin \left(\theta_{1}-\theta_{2}-\frac{\alpha_{1}}{2}\right)\right] .
\end{aligned}
$$

Using $\dot{g}_{N}=\mathbf{W}_{N}^{T} \mathbf{q}$, calculate the normal constrained Jacobian matrix, $\mathbf{W}_{N}^{T}$, as follows:

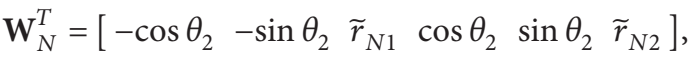

$$
\begin{aligned}
& \widetilde{r}_{N 1}=\left(R+\sigma_{1}\right) \cos \left(\theta_{1}-\theta_{2}\right)-d_{m 1} \cdot \cos \left(\theta_{1}-\theta_{2}-\frac{\alpha_{1}}{2}\right), \\
& \widetilde{r}_{N 2}=\left(x_{1}-x_{2}-\delta\right) \sin \theta_{2}+\left(y_{2}-y_{1}\right) \cos \theta_{2} \\
& -\left(R+\sigma_{1}\right) \cos \left(\theta_{1}-\theta_{2}\right) \\
& +d_{m 2} \cdot \cos \left(\theta_{2}+\frac{\alpha_{2}}{2}\right)-d_{m 1} \\
& \cdot\left[\cos \left(\theta_{2}+\frac{\alpha_{1}}{2}\right)+\cos \left(\theta_{1}-\theta_{2}-\frac{\alpha_{1}}{2}\right)\right] \text {. }
\end{aligned}
$$

3.1. Frictionless Impact Analysis. Since our model is composed of rigid bodies, we can convert the full-edge impact (Figure 5) at the contact of the two sides into a two-point impact. When a two-point impact occurs, we know that $\mathbf{n}_{1}^{T} \mathbf{t}_{2}=0$ or $\mathbf{n}_{2}^{T} \mathbf{t}_{1}=0$. Further, set $\sin \left(\theta_{1}-\theta_{2}\right)=0$, without loss of generality. For this model, it is assumed that $\theta_{1}^{-}=\theta_{2}^{-}=0$.

Matrix $\mathbf{W}_{N}$ can be simplified using equations (11) and (14):

$$
\mathbf{W}_{N}^{T}=\left[\begin{array}{l}
\mathbf{W}_{N 1}^{T} \\
\mathbf{W}_{N 2}^{T}
\end{array}\right]=\left[\begin{array}{llllll}
-1 & 0 & r_{1} & 1 & 0 & r_{3} \\
-1 & 0 & r_{2} & 1 & 0 & r_{4}
\end{array}\right],
$$

where $r_{1}, r_{2}, r_{3}$, and $r_{4}$ are distances shown in Figure 3, which can be calculated as follows:

$$
\begin{aligned}
& r_{1}=\left|R+\frac{W}{2}-d_{m 1} \cdot \cos \frac{\alpha_{1}}{2}\right|, \\
& r_{2}=\left|R-\frac{W}{2}-d_{m 1} \cdot \cos \frac{\alpha_{1}}{2}\right|, \\
& r_{3}=\left|R+\frac{W}{2}-d_{m 2} \cdot \cos \frac{\alpha_{2}}{2}\right|, \\
& r_{4}=\left|R-\frac{W}{2}-d_{m 2} \cdot \cos \frac{\alpha_{2}}{2}\right| .
\end{aligned}
$$

We then calculate $\mathbf{G}_{N N}$ and $\mathbf{G}_{N N}^{-1}$ as follows: 


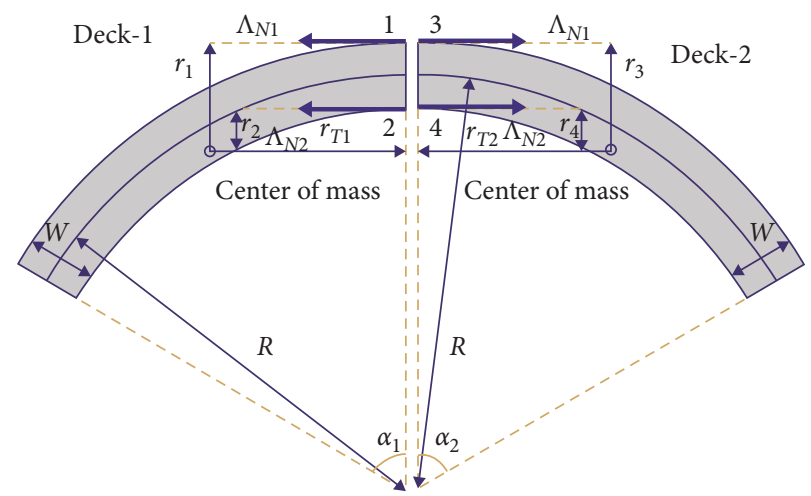

Figure 5: Full-edge frictionless collision between deck-1 and deck-2.

$$
\begin{aligned}
& \mathbf{G}_{N N}=\mathbf{W}_{N}^{T} \mathbf{M}^{-1} \mathbf{W}_{N}=\left(\begin{array}{cc}
\frac{1}{m_{1}}+\frac{1}{m_{2}}+\frac{r_{1}^{2}}{I_{1}}+\frac{r_{3}^{2}}{I_{2}} & \frac{1}{m_{1}}+\frac{1}{m_{2}}+\frac{r_{1} r_{2}}{I_{1}}+\frac{r_{3} r_{4}}{I_{2}} \\
\frac{1}{m_{1}}+\frac{1}{m_{2}}+\frac{r_{1} r_{2}}{I_{1}}+\frac{r_{3} r_{4}}{I_{2}} & \frac{1}{m_{1}}+\frac{1}{m_{2}}+\frac{r_{2}^{2}}{I_{1}}+\frac{r_{4}^{2}}{I_{2}}
\end{array}\right), \\
& \mathbf{G}_{N N}^{-1}=\frac{1}{\left|\mathbf{G}_{N N}\right|}=\left(\begin{array}{cc}
\frac{1}{m_{1}}+\frac{1}{m_{2}}+\frac{r_{2}^{2}}{I_{1}}+\frac{r_{4}^{2}}{I_{2}}-\left(\frac{1}{m_{1}}+\frac{1}{m_{2}}+\frac{r_{1} r_{2}}{I_{1}}+\frac{r_{3} r_{4}}{I_{2}}\right) \\
-\left(\frac{1}{m_{1}}+\frac{1}{m_{2}}+\frac{r_{1} r_{2}}{I_{1}}+\frac{r_{3} r_{4}}{I_{2}}\right) & \frac{1}{m_{1}}+\frac{1}{m_{2}}+\frac{r_{1}^{2}}{I_{1}}+\frac{r_{3}^{2}}{I_{2}}
\end{array}\right) .
\end{aligned}
$$

According to Newton's impact law [25], the normal impulse, $\Lambda_{N}$, is defined as follows:

$$
\boldsymbol{\Lambda}_{N}=\left(\begin{array}{c}
\Lambda_{N 1} \\
\Lambda_{N 2}
\end{array}\right)=-\mathbf{G}_{N N}^{-1}\left(\mathbf{I}_{2}+\overline{\bar{\varepsilon}}_{N}\right) \dot{g}_{N}^{-} .
$$

In this equation, $\mathbf{I}_{2}$ is an identity matrix and the Newton impact coefficients of the two impact points are the same, $\varepsilon_{N 1}=\varepsilon_{N 2}=\varepsilon_{N}$. We then decompose the above matrix as follows:

$$
\begin{aligned}
& \Lambda_{N 1}=-\frac{\dot{g}_{N}^{-}}{\left|G_{N N}\right|} \cdot\left(1+\varepsilon_{N}\right)\left(r_{2} \frac{r_{2}-r_{1}}{I_{1}}+r_{4} \frac{r_{4}-r_{3}}{I_{2}}\right), \\
& \Lambda_{N 2}=-\frac{\dot{g}_{N}^{-}}{\left|G_{N N}\right|} \cdot\left(1+\varepsilon_{N}\right)\left(r_{1} \frac{r_{1}-r_{2}}{I_{1}}+r_{3} \frac{r_{3}-r_{4}}{I_{2}}\right) .
\end{aligned}
$$

Since $\left(1+\varepsilon_{N}\right)>0,\left|\mathbf{G}_{N N}\right|>0$, and the contact occurs if and only if $\dot{g}_{N}^{-}<0$, one obtains the following:

$$
\begin{aligned}
& \Lambda_{N 1}>0 \Longleftrightarrow r_{2} \frac{r_{2}-r_{1}}{I_{1}}+r_{4} \frac{r_{4}-r_{3}}{I_{2}}>0, \\
& \Lambda_{N 2}>0 \Longleftrightarrow r_{1} \frac{r_{1}-r_{2}}{I_{1}}+r_{3} \frac{r_{3}-r_{4}}{I_{2}}>0 .
\end{aligned}
$$

In order to prevent the main girders from rotating during full-edge impact, equations (21) and (22) must be satisfied at the same time, so that both $\Lambda_{N 1}$ and $\Lambda_{N 2}$ are positive. After examining all the cases of $r_{1}, r_{2}, r_{3}$, and $r_{4}$ in equation (17), it is concluded that only the following two cases can satisfy that both $\Lambda_{N 1}$ and $\Lambda_{N 2}$ are positive. In the following analysis, two variables, $s_{i}$ and $s_{j}$, which are only related to geometric parameters, are introduced for the convenience of analyzing the following two cases:

Case 1. $-(W / 2)<s_{i}<(W / 2), s_{j}<-(W / 2)$

$$
\begin{aligned}
& \frac{2 s_{i} \cdot \alpha_{j} \cdot\left((W / 2)+s_{i}\right)}{W \cdot \alpha_{i} \cdot\left((W / 2)+s_{j}\right)}<-1, \\
& \frac{2 s_{i} \cdot \alpha_{j} \cdot\left((W / 2)-s_{i}\right)}{W \cdot \alpha_{i} \cdot\left((W / 2)-s_{j}\right)}<1 .
\end{aligned}
$$

Case 2. $-(W / 2)<s_{i}<(W / 2),-(W / 2)<s_{j}<(W / 2)$

$$
\begin{aligned}
& \frac{s_{i} \cdot \alpha_{j} \cdot\left((W / 2)+s_{i}\right)}{s_{j} \cdot \alpha_{i} \cdot\left((W / 2)+s_{j}\right)}<-1, \\
& \frac{s_{i} \cdot \alpha_{j} \cdot\left((W / 2)-s_{i}\right)}{s_{j} \cdot \alpha_{i} \cdot\left((W / 2)-s_{j}\right)}>-1 .
\end{aligned}
$$

For these cases, $s_{i}=d_{m i} \cdot \cos \left(\alpha_{i} / 2\right)-R$ and $s_{j}=d_{m j} \cdot \cos \left(\alpha_{j} / 2\right)-R$, where $s_{i}$ and $s_{j}$ represent deck-1 and deck-2, respectively. According to equations (21) and (22), it can be concluded that the different contact conditions are determined by the geometric characteristics and 
inertial conditions of the bridge segments. This conclusion can be used to guide engineering practice.

\section{Frictional Impact Analysis}

When multiple points with frictional contact impacts are considered, the traditional approach is to consider the normal and tangential impulses of all possible contacts. For this analysis, $\Lambda_{N}=\left\{\Lambda_{N i}\right\}$ and $\Lambda_{T}=\left\{\Lambda_{T i}\right\}$, but $\mathbf{G}_{N T}, \mathbf{G}_{T N}$, and $\mathbf{G}_{T T}$ are all singular matrices. In this paper, we use the alternative description of multipoint impact proposed by Dimitrakopoulos [27] (Figure 6) to solve the overconstrained problem of multibody dynamic impact and therefore avoid the closed solutions of all singular points.

Using the results of Dimitrakopoulos [27], we can calculate $\mathbf{W}_{T}^{T}, \mathbf{G}_{N T}, \mathbf{G}_{T N}$, and $G_{T T}$ as follows:

$$
\begin{aligned}
& \mathbf{W}_{T}^{T}=\left(\begin{array}{cccccc}
0 & 1 & r_{T 1} & 0 & -1 & r_{T 2} \\
0 & 1 & r_{T 1} & 0 & -1 & r_{T 2}
\end{array}\right) \text {, } \\
& \Lambda_{T 1}+\Lambda_{T 2}=\mu \Lambda_{N 1}+\mu \Lambda_{N 2}=\left(\begin{array}{ll}
\mu & \mu
\end{array}\right)\left(\begin{array}{c}
\Lambda_{N 1} \\
\Lambda_{N 2}
\end{array}\right)=\overline{\bar{\mu}} \boldsymbol{\Lambda}_{N}=\boldsymbol{\Lambda}_{T}, \\
& \mathbf{G}_{N T}=\mathbf{W}_{N}^{T} \mathbf{M}^{-1} \mathbf{W}_{T}=\left(\begin{array}{l}
\frac{r_{1} r_{T 1}}{I_{1}}+\frac{r_{3} r_{T 2}}{I_{2}} \\
\frac{r_{2} r_{T 1}}{I_{1}}+\frac{r_{4} r_{T 2}}{I_{2}}
\end{array}\right)=\mathbf{G}_{T N}^{T}, \\
& G_{T T}=\mathbf{W}_{T}^{T} \mathbf{M}^{-1} \mathbf{W}_{T}=\frac{1}{m_{1}}+\frac{1}{m_{2}}+\frac{r_{T 1}^{2}}{I_{1}}+\frac{r_{T 2}^{2}}{I_{2}} .
\end{aligned}
$$

Because of the existence of matrices $\mathbf{G}_{N T}, \mathbf{G}_{T N}$, and $\mathbf{G}_{T T}$ [27], one can avoid the tedious calculations caused by irreversibility, and the discussion of the subsequent analysis of frictional impact can proceed. All the possible cases of slip and stick, when the two furthest points of each deck are in contact with the other deck contour, are illustrated in Figure 7.

\subsection{Multipoint Impact}

4.1.1. Deck-1 Slides down Relative to Deck-2. When deck-1 slides down relative to deck-2, $\Lambda_{N 1}>0, \Lambda_{N 2}>0 v_{N 1}=$ $v_{N 2}=v_{T R 1}=v_{T R 2}=0, \Lambda_{T R 1}=2 \overline{\bar{\mu}} \Lambda_{N 1}, \Lambda_{T R 2}=2 \overline{\bar{\mu}} \Lambda_{N 2}$, and $\Lambda_{T L 1}=\Lambda_{T L 2}=0$.

It follows from equation (4) that the impulses in the normal direction are as follows:

$$
\begin{aligned}
& \boldsymbol{\Lambda}_{N}=-\left(\mathbf{G}_{N N}+\mathbf{G}_{N T} \overline{\bar{\mu}}\right)^{-1}\left(\overline{\bar{\varepsilon}}_{N}+\mathbf{E}\right) \dot{\mathfrak{g}}_{N}^{-}, \\
& \mathbf{v}_{T L}=-\left(\mathbf{G}_{T N}+\mathbf{G}_{T T} \overline{\bar{\mu}}\right) \boldsymbol{\Lambda}_{N}-\mathbf{E} \dot{g}_{T}^{-} .
\end{aligned}
$$
obtains
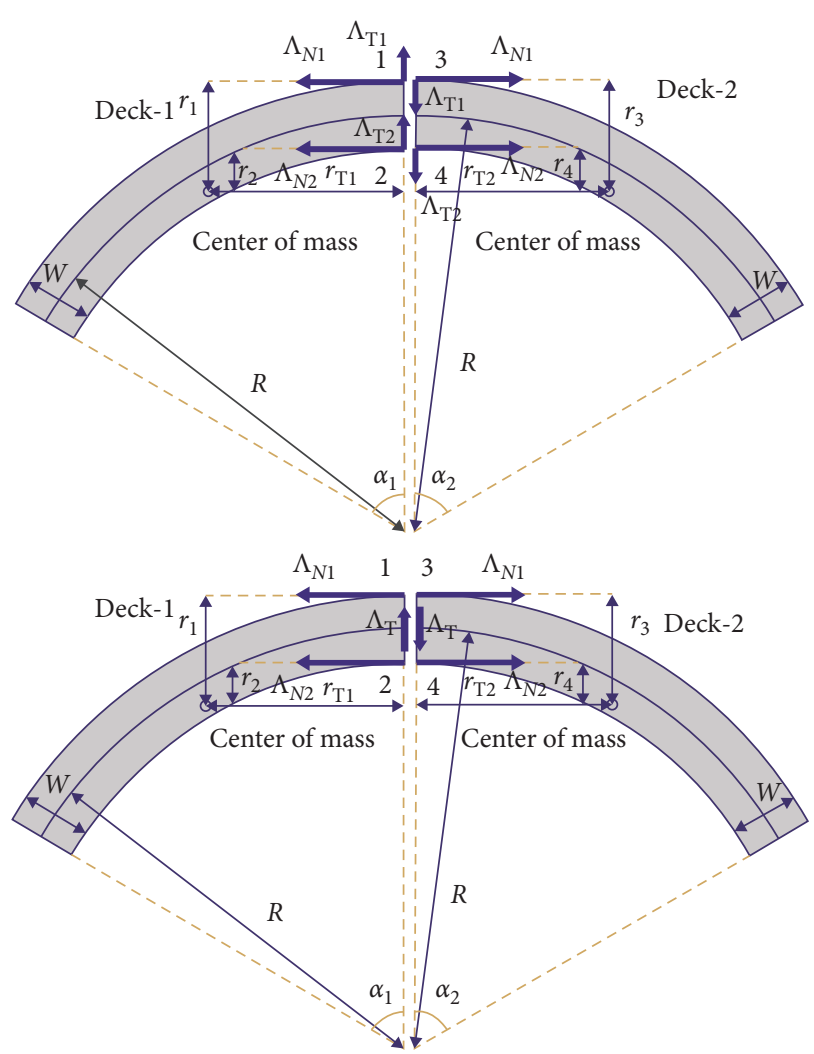

FIgURE 6: Full-edge frictional impact between deck-1 and deck-2.

$$
\mathbf{M}\left(\mathbf{u}^{+}-\mathbf{u}^{-}\right)=\mathbf{W}_{N} \boldsymbol{\Lambda}_{N}+\mathbf{W}_{T} \boldsymbol{\Lambda}_{T} .
$$

From equations (27) and (28), we have

$$
\mathbf{u}^{+}=\mathbf{u}^{-}-\mathbf{M}^{-1}\left(\mathbf{W}_{N}+\overline{\overline{\boldsymbol{\mu}}} \mathbf{W}_{T}\right)\left(\mathbf{G}_{N N}+\mathbf{G}_{N T} \overline{\overline{\boldsymbol{\mu}}}\right)^{-1}\left(\boldsymbol{\varepsilon}_{N}+\mathbf{E}\right) \dot{\boldsymbol{g}}_{N}^{-} .
$$

From equation (4), we know that $\dot{g}_{T}^{+}=\mathbf{v}_{T R}-$ $\mathbf{v}_{T L}=-\mathbf{v}_{T L} \leq 0$. The preimpact tangential normal velocity rate is denoted as $\eta$ and plays an important role in the analysis of the following cases. Using this inequality, we have

$$
\begin{aligned}
\eta= & \frac{\dot{g}_{T}^{-}}{\dot{g}_{N}^{-}} \geq \frac{1+\varepsilon_{N}}{\left|\mathbf{G}_{N N}+\mathbf{G}_{N T} \overline{\overline{\boldsymbol{\mu}}}\right|} \\
& \cdot \frac{W^{2}}{I_{1} I_{2}} \cdot\left\{\begin{array}{c}
\frac{\mu\left(I_{1}+I_{2}\right)\left(m_{1}+m_{2}\right)}{m_{1} m_{2}}+\left(r_{T 1}-r_{T 2}\right) \\
{\left[\mu r_{T 1}-\mu r_{T 2}+\frac{1}{W}\left(r_{2} r_{3}-r_{1} r_{4}\right)\right]}
\end{array}\right\} \\
= & \eta_{\max } .
\end{aligned}
$$

4.1.2. Deck-1 Slides up Relative to Deck-2. When deck-1 slides up relative to deck-2, $\Lambda_{N 1}>0, \Lambda_{N 2}>0, v_{N 1}=v_{N 2}=$ $v_{T L 1}=v_{T L 2}=0, \quad \Lambda_{T L 1}=2 \overline{\bar{\mu}} \Lambda_{N 1}, \quad \Lambda_{T L 2}=2 \overline{\bar{\mu}} \Lambda_{N 2}, \quad$ and $\Lambda_{T R 1}=\Lambda_{T R 2}=0$.

It follows from equation (4) that the impulses in the normal direction are as follows: 


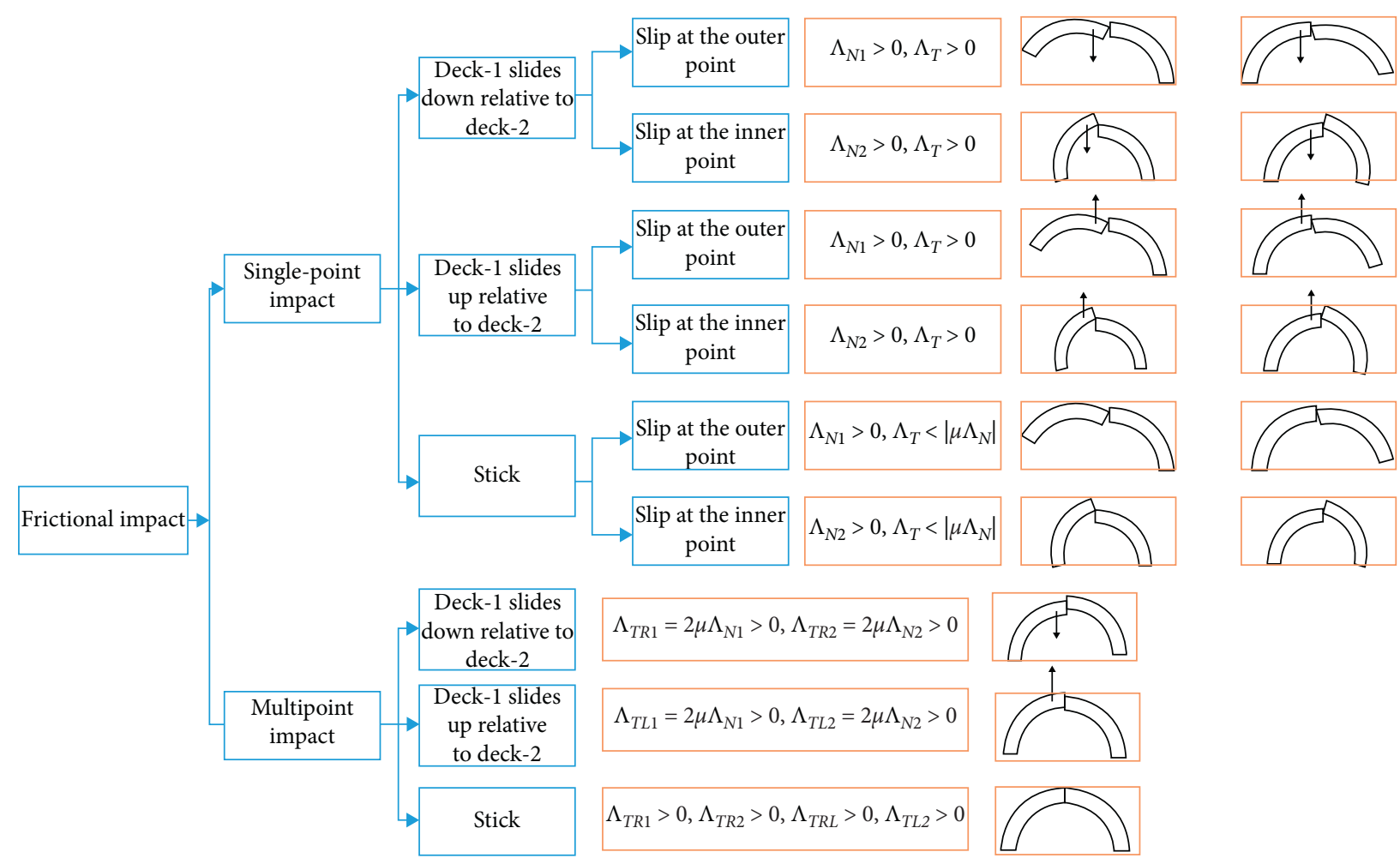

Figure 7: Classification of different frictional postimpact states of the curved bridge.

$$
\boldsymbol{\Lambda}_{N}=-\left(1+\varepsilon_{N}\right) \dot{g}_{N}^{-}\left(\mathbf{G}_{N N}-\mathbf{G}_{N T} \overline{\bar{\mu}}\right)^{-1}\left(\begin{array}{l}
1 \\
1
\end{array}\right)
$$

From equations (28) and (31), we have

$$
\mathbf{u}^{+}=\mathbf{u}^{-}-\mathbf{M}^{-1}\left(\mathbf{W}_{N}-\overline{\bar{\mu}} \mathbf{W}_{T}\right)\left(\mathbf{G}_{N N}-\mathbf{G}_{N T} \overline{\bar{\mu}}\right)^{-1}\left(\boldsymbol{\varepsilon}_{N}+\mathbf{E}\right) \dot{g}_{N}^{-} .
$$

From equation (4), we know that $\dot{g}_{T}^{+}=\mathbf{v}_{T R^{-}}$ $\mathbf{v}_{T L}=-\mathbf{v}_{T L} \leq 0$ and obtain the following:

$$
\begin{aligned}
\eta= & \frac{\dot{g}_{T}^{-}}{\dot{g}_{N}^{-}} \leq \frac{1+\varepsilon_{N}}{\left|\mathbf{G}_{N N}-\mathbf{G}_{N T} \overline{\bar{\mu}}\right|} \\
& \cdot \frac{W^{2}}{I_{1} I_{2}} \cdot\left\{\begin{array}{c}
\frac{\mu\left(I_{1}+I_{2}\right)\left(m_{1}+m_{2}\right)}{m_{1} m_{2}}+\left(r_{T 1}-r_{T 2}\right) \\
{\left[\mu r_{T 1}-\mu r_{T 2}+\frac{1}{W}\left(r_{1} r_{4}-r_{2} r_{3}\right)\right]}
\end{array}\right\} \\
= & \eta_{\min } .
\end{aligned}
$$

4.1.3. Stick. When deck- 1 sticks to deck- $2, \Lambda_{N 1}>0, \Lambda_{N 2}>0$, and $\left|\Lambda_{T 1}\right|<\mu \Lambda_{N 1}, \quad\left|\Lambda_{T 2}\right|<\mu \Lambda_{N 2}, \quad v_{N 1}=v_{N 2}=0$, and the following constraints can be obtained:

$$
\begin{aligned}
& \Lambda_{T R 1}=\mu \Lambda_{N 1}+\Lambda_{T 1}>0, \\
& \Lambda_{T R 2}=\mu \Lambda_{N 2}+\Lambda_{T 2}>0, \\
& \Lambda_{T L 1}=\mu \Lambda_{N 1}-\Lambda_{T 1}>0, \\
& \Lambda_{T L 2}=\mu \Lambda_{N 2}-\Lambda_{T 2}>0 .
\end{aligned}
$$

Then, we have $v_{T L 1}=v_{T L 2}=v_{T R 1}=v_{T R 2}=0$. It follows from equation (4) that the impulses in the normal and tangential direction are as follows:

$$
\begin{array}{r}
\left(\mathbf{G}_{N N}-\mathbf{G}_{N T} \overline{\bar{\mu}}\right) \boldsymbol{\Lambda}_{N}+\mathbf{G}_{N T} \boldsymbol{\Lambda}_{T R}+\left(\overline{\bar{\varepsilon}}_{\mathbf{N}}+\mathbf{E}\right) \dot{g}_{T}^{-}=0, \\
\left(\mathbf{G}_{T N}-\mathbf{G}_{T T} \overline{\bar{\mu}}\right) \boldsymbol{\Lambda}_{N}+\mathbf{G}_{T T} \boldsymbol{\Lambda}_{T R}+\mathbf{E} \cdot \dot{g}_{T}^{-}=0 .
\end{array}
$$

From the above analysis, we list the conditions for the occurrence of the multipoint stick and multipoint slip using the preimpact tangential normal velocity rate, $\eta$ :

(1) When deck-1 slides down relative to deck-2: $\eta \geq \eta_{\max }$

(2) When deck-1 slides up relative to deck-2: $\eta \leq \eta_{\min }$

(3) When deck-1 sticks to deck-2: $\eta_{\min } \leq \eta \leq \eta_{\max }$ where 


$$
\begin{aligned}
& \eta_{\min }=\frac{1+\varepsilon_{N}}{\left|\mathbf{G}_{N N}-\mathbf{G}_{N T} \overline{\overline{\mathbf{\mu}}}\right|} \cdot \frac{W^{2}}{I_{1} I_{2}} \cdot\left\{\frac{\mu\left(I_{1}+I_{2}\right)\left(m_{1}+m_{2}\right)}{m_{1} m_{2}}+\left(r_{T 1}-r_{T 2}\right) \cdot\left[\mu r_{T 1}-\mu r_{T 2}+\frac{1}{W}\left(r_{1} r_{4}-r_{2} r_{3}\right)\right]\right\}, \\
& \eta_{\max }=\frac{1+\varepsilon_{N}}{\left|\mathbf{G}_{N N}+\mathbf{G}_{N T} \overline{\overline{\mathbf{\mu}}}\right|} \cdot \frac{W^{2}}{I_{1} I_{2}} \cdot\left\{\frac{\mu\left(I_{1}+I_{2}\right)\left(m_{1}+m_{2}\right)}{m_{1} m_{2}}+\left(r_{T 1}-r_{T 2}\right) \cdot\left[\mu r_{T 1}-\mu r_{T 2}+\frac{1}{W}\left(r_{2} r_{3}-r_{1} r_{4}\right)\right]\right\} .
\end{aligned}
$$

Two conclusions can be drawn from the above analysis. First, from equations (21) and (22), it can be concluded that the different contact conditions are determined by the geometric characteristics and inertial parameters of the bridge segments. Secondly, according to equation (36), the preimpact tangential normal velocity rate, $\eta$, the normal impact recovery coefficient, $\varepsilon_{N}$, and the transverse friction coefficient, $\mu$, determine the state of motion between the decks after contact.

\subsection{Single-Point Frictional Impact Analysis}

4.2.1. Stick or Slip at the Outer Point of Bridge Segments. When deck-1 slides down relative to deck-2, the following can be obtained from equation (30):

$$
\eta=\frac{\dot{g}_{T}^{-}}{\dot{g}_{N}^{-}} \geq \frac{\mu\left(\left(m_{1}+m_{2}\right) / m_{1} m_{2}\right)+\left(\left(\left(r_{1} r_{T 1}+\mu r_{T 1}^{2}\right)\right) / I_{1}\right)+\left(\left(r_{3} r_{T 2}+\mu r_{T 2}^{2}\right) / I_{2}\right)}{\left(\left(m_{1}+m_{2}\right) / m_{1} m_{2}\right)+\left(\left(r_{1}^{2}-\mu r_{1} r_{T 1}\right) / I_{1}\right)+\left(\left(r_{3}^{2}-\mu r_{3} r_{T 2}\right) / I_{2}\right)} \times\left(1+\varepsilon_{N}\right)=\eta_{\max } .
$$

When deck-1 slides up relative to deck-2, we can obtain from equation (33) the following:

$$
\eta=\frac{\dot{g}_{T}^{-}}{\dot{g}_{N}^{-}} \leq \frac{\mu\left(\left(m_{1}+m_{2}\right) / m_{1} m_{2}\right)+\left(\left(r_{1} r_{T 1}-\mu r_{T 1}^{2}\right) / I_{1}\right)+\left(\left(r_{3} r_{T 2}-\mu r_{T 2}^{2}\right) / I_{2}\right)}{\left(m_{1} m_{2} / m_{1} m_{2}\right)+\left(\left(r_{1}^{2}-\mu r_{1} r_{T 1}\right) / I_{1}\right)+\left(\left(r_{3}^{2}-\mu r_{3} r_{T 2}\right) / I_{2}\right)} \times\left(1+\varepsilon_{N}\right)=\eta_{\text {min }} .
$$

According to the above analyses, we list the conditions for the occurrence of the single-point stick and single-point slip at the outer point of the bridge segments, respectively, based on the preimpact tangential normal velocity rate, $\eta$ :

(1) When deck-1 slides down relative to deck-2: $\eta \geq \eta_{\max }$
(2) When deck-1 slides up relative to deck-2: $\eta \leq \eta_{\text {min }}$ (3) When deck-1 sticks to deck-2: $\eta_{\min } \leq \eta \leq \eta_{\max }$ where

$$
\begin{aligned}
& \eta_{\min }=\frac{\mu\left(\left(m_{1}+m_{2}\right) / m_{1} m_{2}\right)+\left(\left(r_{1} r_{T 1}-\mu r_{T 1}^{2}\right) / I_{1}\right)+\left(\left(r_{3} r_{T 2}-\mu r_{T 2}^{2}\right) / I_{2}\right)}{\left(\left(m_{1}+m_{2}\right) / m_{1} m_{2}\right)+\left(\left(r_{1}^{2}-\mu r_{1} r_{T 1}\right) / I_{1}\right)+\left(\left(r_{3}^{2}-\mu r_{3} r_{T 2}\right) / I_{2}\right)} \times\left(1+\varepsilon_{N}\right), \\
& \eta_{\max }=\frac{\mu\left(\left(m_{1}+m_{2}\right) / m_{1} m_{2}\right)+\left(\left(r_{1} r_{T 1}+\mu r_{T 1}^{2}\right) / I_{1}\right)+\left(\left(r_{3} r_{T 2}+\mu r_{T 2}^{2}\right) / I_{2}\right)}{\left(\left(m_{1}+m_{2}\right) / m_{1} m_{2}\right)+\left(\left(r_{1}^{2}-\mu r_{1} r_{T 1}\right) / I_{1}\right)+\left(\left(r_{3}^{2}-\mu r_{3} r_{T 2}\right) / I_{2}\right)} \times\left(1+\varepsilon_{N}\right) .
\end{aligned}
$$

4.2.2. Stick or Slip at the Inner Point of Bridge Segments.

When deck-1 slides down relative to deck-2, we can obtain from equation (30) the following:

$$
\eta=\frac{\dot{g}_{T}^{-}}{\dot{g}_{N}^{-}} \geq \frac{\mu\left(\left(m_{1}+m_{2}\right) / m_{1} m_{2}\right)+\left(\left(r_{2} r_{T 1}+\mu r_{T 1}^{2}\right) / I_{1}\right)+\left(\left(r_{4} r_{T 2}+\mu r_{T 2}^{2}\right) / I_{2}\right)}{\left(\left(m_{1}+m_{2}\right) / m_{1} m_{2}\right)+\left(\left(r_{2}^{2}-\mu r_{2} r_{T 1}\right) / I_{1}\right)+\left(\left(r_{4}^{2}-\mu r_{4} r_{T 2}\right) / I_{2}\right)} \times\left(1+\varepsilon_{N}\right)=\eta_{\max } .
$$



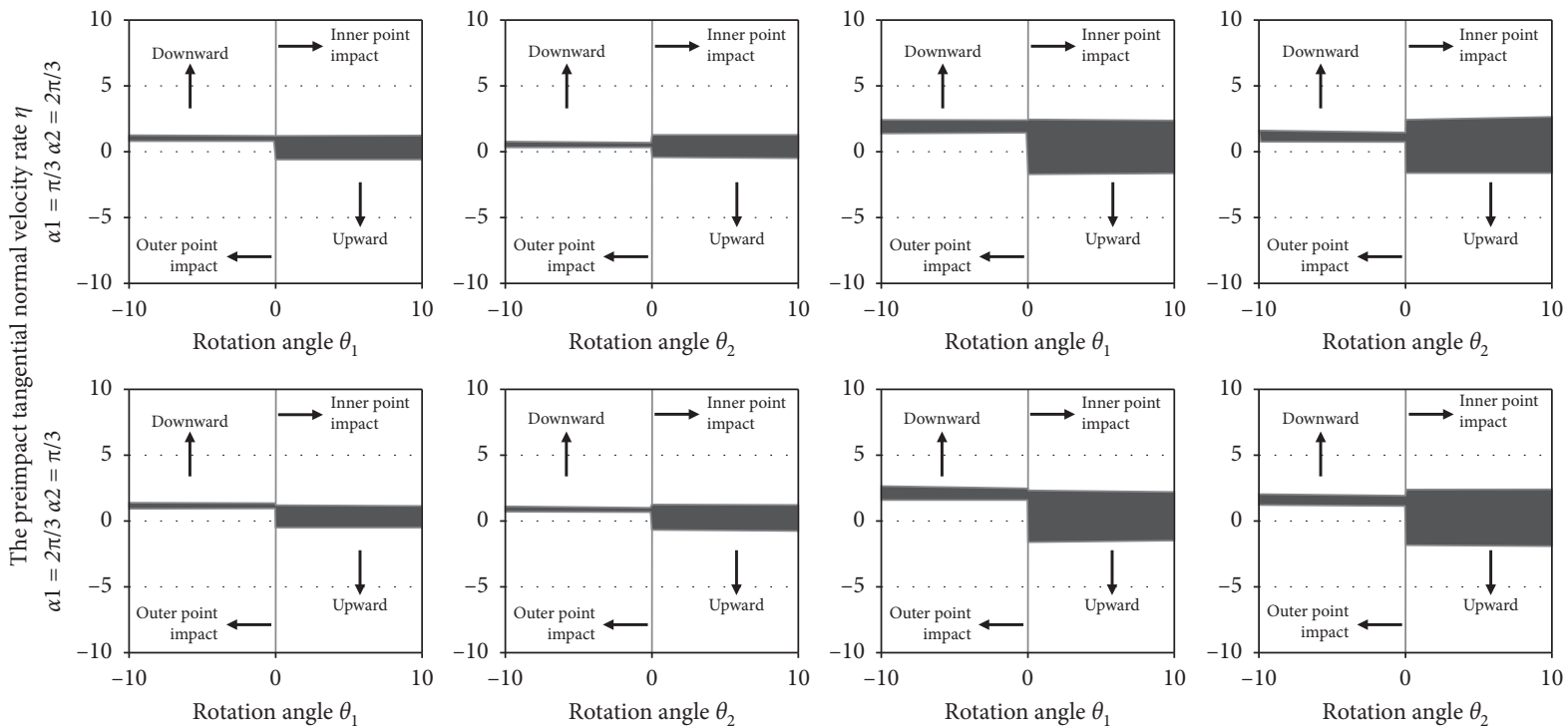

Figure 8: Identification of stick and upward and downward slip states for different preimpact transverse and normal velocity rates for various preimpact rotations of center angle of girder at $60^{\circ}$ and $120^{\circ}$.

When deck-1 slides up relative to deck-2, we can obtain from equation (33) the following:

$$
\eta=\frac{\dot{g}_{T}^{-}}{\dot{g}_{N}^{-}} \leq \frac{-\mu\left(\left(m_{1}+m_{2}\right) / m_{1} m_{2}\right)+\left(\left(r_{2} r_{T 1}-\mu r_{T 1}^{2}\right) / I_{1}\right)+\left(\left(r_{4} r_{T 2}-\mu r_{T 2}^{2}\right) / I_{2}\right)}{\left(\left(m_{1}+m_{2}\right) / m_{1} m_{2}\right)+\left(\left(r_{2}^{2}-\mu r_{2} r_{T 1}\right) / I_{1}\right)+\left(\left(r_{4}^{2}-\mu r_{4} r_{T 2}\right) / I_{2}\right)} \times\left(1+\varepsilon_{N}\right)=\eta_{\min } .
$$

According to the above analyses, we list the conditions for the occurrence of the single-point stick and single-point slip at the inner point of the bridge segments, respectively, based on the preimpact tangential normal velocity rate, $\eta$ :

(1) When deck-1 slides down relative to deck-2: $\eta \geq \eta_{\max }$
(2) When deck-1 slides up relative to deck-2: $\eta \leq \eta_{\text {min }}$

(3) When deck-1 sticks to deck-2: $\eta_{\min } \leq \eta \leq \eta_{\max }$

$$
\begin{aligned}
\eta_{\max } & =\frac{\mu\left(\left(m_{1}+m_{2}\right) / m_{1} m_{2}\right)+\left(\left(r_{2} r_{T 1}+\mu r_{T 1}^{2}\right) / I_{1}\right)+\left(\left(r_{4} r_{T 2}+\mu r_{T 2}^{2}\right) / I_{2}\right)}{\left(\left(m_{1}+m_{2}\right) / m_{1} m_{2}\right)+\left(\left(r_{2}^{2}-\mu r_{2} r_{T 1}\right) / I_{1}\right)+\left(\left(r_{4}^{2}-\mu r_{4} r_{T 2}\right) / I_{2}\right)} \times\left(1+\varepsilon_{N}\right), \\
\eta_{\min } & =\frac{-\mu\left(\left(m_{1}+m_{2}\right) / m_{1} m_{2}\right)+\left(\left(r_{2} r_{T 1}-\mu r_{T 1}^{2}\right) / I_{1}\right)+\left(\left(r_{4} r_{T 2}-\mu r_{T 2}^{2}\right) / I_{2}\right)}{\left(\left(m_{1}+m_{2}\right) / m_{1} m_{2}\right)+\left(\left(r_{2}^{2}-\mu r_{2} r_{T 1}\right) / I_{1}\right)+\left(\left(r_{4}^{2}-\mu r_{4} r_{T 2}\right) / I_{2}\right)} \times\left(1+\varepsilon_{N}\right) .
\end{aligned}
$$

From equations (39), (40), (43), and (44), it is concluded that the preimpact tangential normal velocity rate, $\eta$, the normal impact recovery coefficient, $\varepsilon_{N}$, and the transverse friction coefficient, $\mu$, determine the state of motion between the decks after contact (sliding upward, sliding downward, or stick). It is necessary to ensure the two decks stick after impact (without relative sliding) to avoid the rotation of the decks due to the impact.

In the following analyses and figures, "upward" refers to deck-1 sliding up relative to deck-2 and "downward" to deck-1 sliding down relative to deck-2. Potential contact points are indicated in Figure 3. A planar rectangular coordinate system is established considering only the rotation of the main girder in the plane. The preimpact tangential normal velocity rate, $\eta$, is calculated by using the parameters modelling the process of impact. In the process of impact, the stick-slip between the main girders is affected not only by the geometric parameters of the main girders but also by the preimpact tangential normal velocity, $\eta$. The influencing geometric factors are the curvature of the two decks, the ratio of width to radius of the main girder, and the rotation angle of the two decks, $\alpha_{i}$. From these parameters, the 

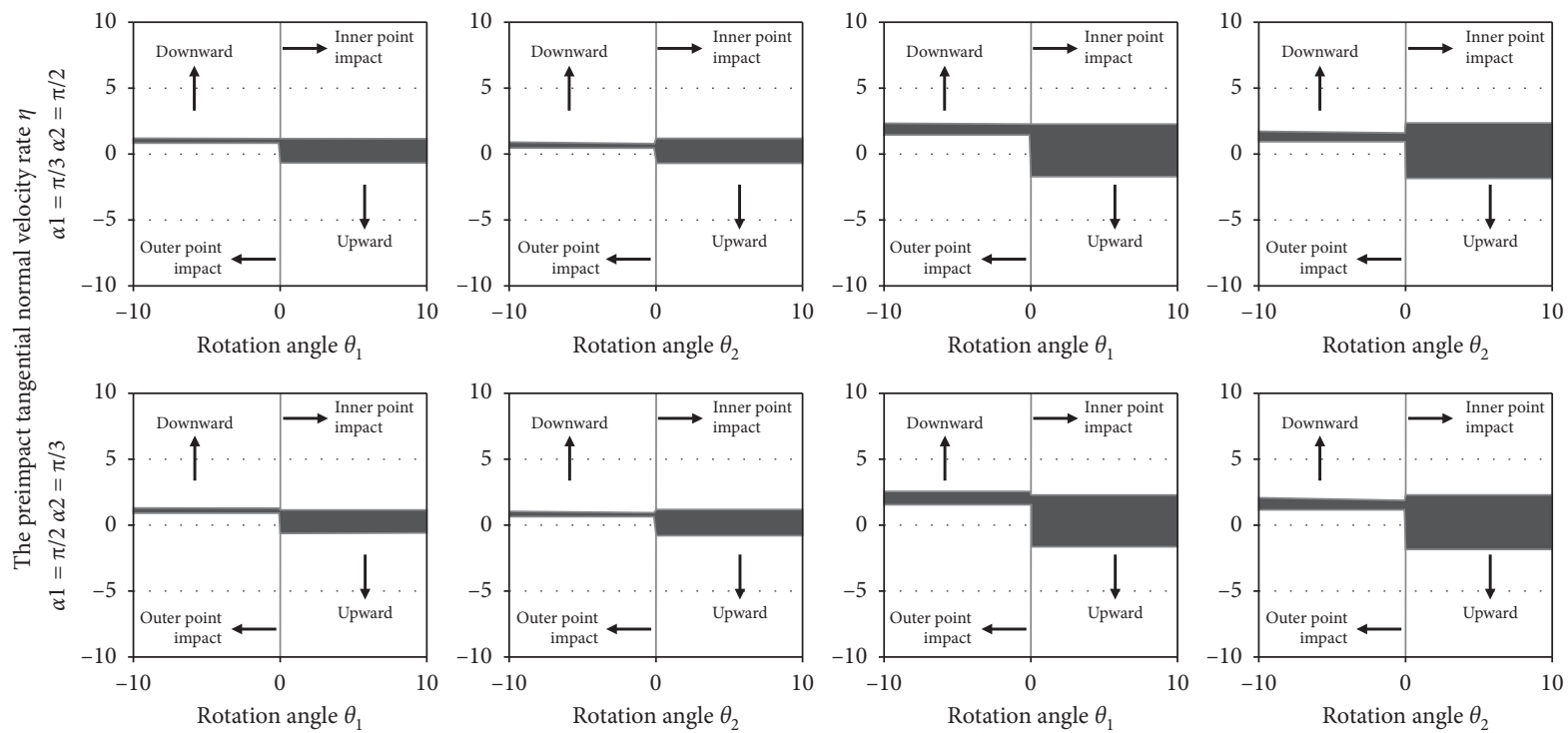

FIGURE 9: Identification of stick and upward and downward slip states for different preimpact transverse and normal velocity rates for various preimpact rotations of the center angle of girder at $60^{\circ}$ and $90^{\circ}$.
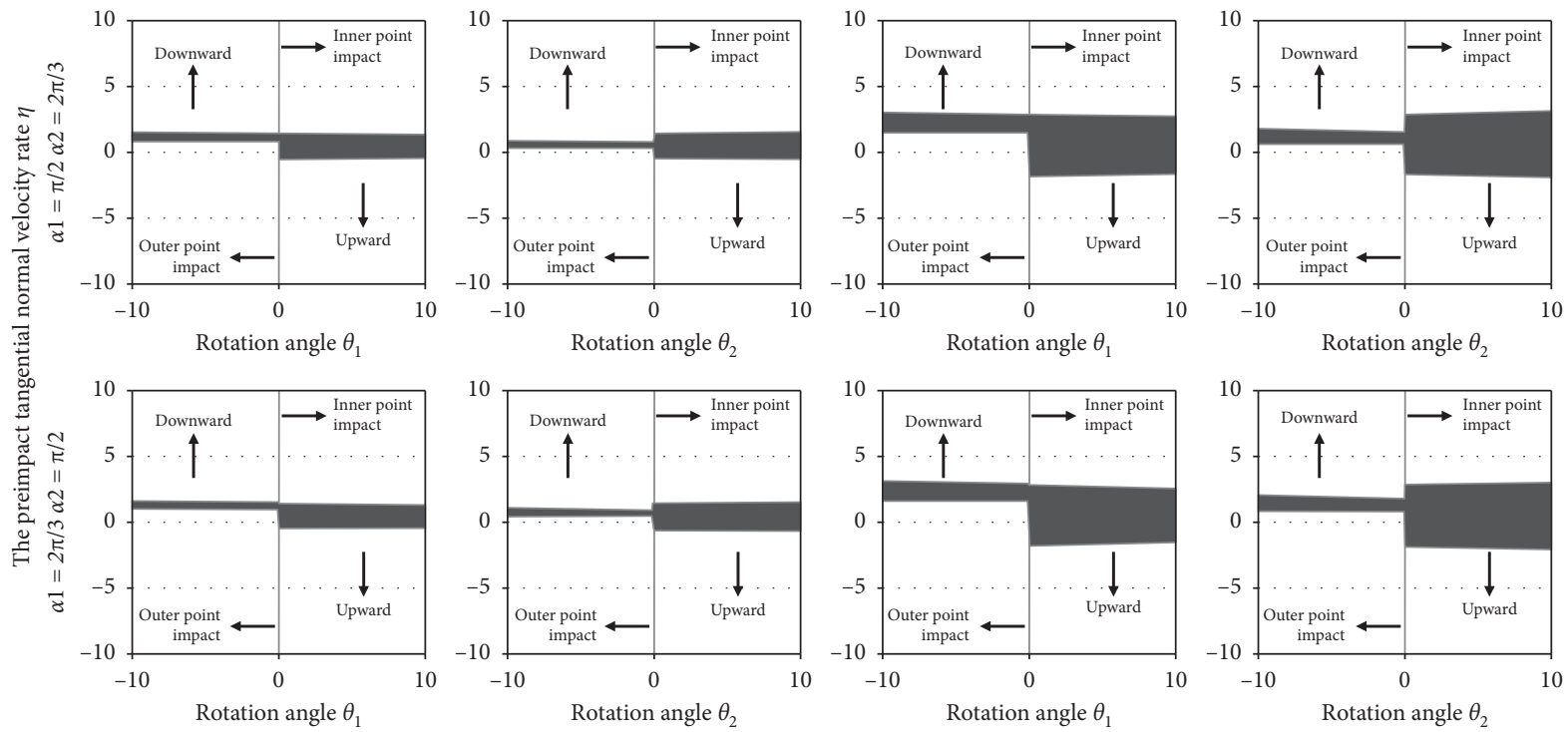

Figure 10: Identification of stick and upward and downward slip states for different preimpact transverse and normal velocity rates for various preimpact rotations of the center angle of girder at $90^{\circ}$ and $120^{\circ}$.

transverse and normal constrained Jacobian matrices can be calculated from the formulas in this paper. Without loss of generality, we assume that the width is $15 \mathrm{~m}$ and the radius is $100 \mathrm{~m}$. The normal recovery coefficient of the main girder is 0.5 , and we assume that the geometry satisfies $R-(W / 2)-$ $d_{m i} \cdot \cos \left(\alpha_{i} / 2\right)>0$. In Figures $8-11$, it is assumed that $\sigma_{1}=$ $(W / 2)$ and $\sigma_{2}=(W / 2)$.

The variables analyzed include the tangential friction coefficient and the center angle of the girders. We assume that the tangential friction coefficient is 0.5 and 1 , respectively. Figures 8-11 show the different center angles of the girders. When a point of one deck contacts the edge of the other deck, this case is called a single-point contact. When friction is considered, the main girder will slip and rotate when the friction exceeds the maximum static sliding friction. Figure 8 is a diagram drawn for the center angles of the girders of $60^{\circ}$ and $120^{\circ}$, respectively. It can be seen from the diagram that, with the increase in friction coefficient, the area of adhesion increases because more force is needed to overcome it.

In Figures 8-11, the area between the upper and lower lines represents the stick condition, $\left[-10^{\circ}, 10^{\circ}\right]$ represents the rotation angle range of the two decks, and $[-10,10]$ represents the range of preimpact tangential normal velocity ratio, $\eta$. The upper and lower parts of the adhesion area in these figures represent different sliding cases, including the inward and outward sliding cases of the inner and outer points of the two main girders. The area and situation of different motion states can clearly be observed in Figures 8-11. 

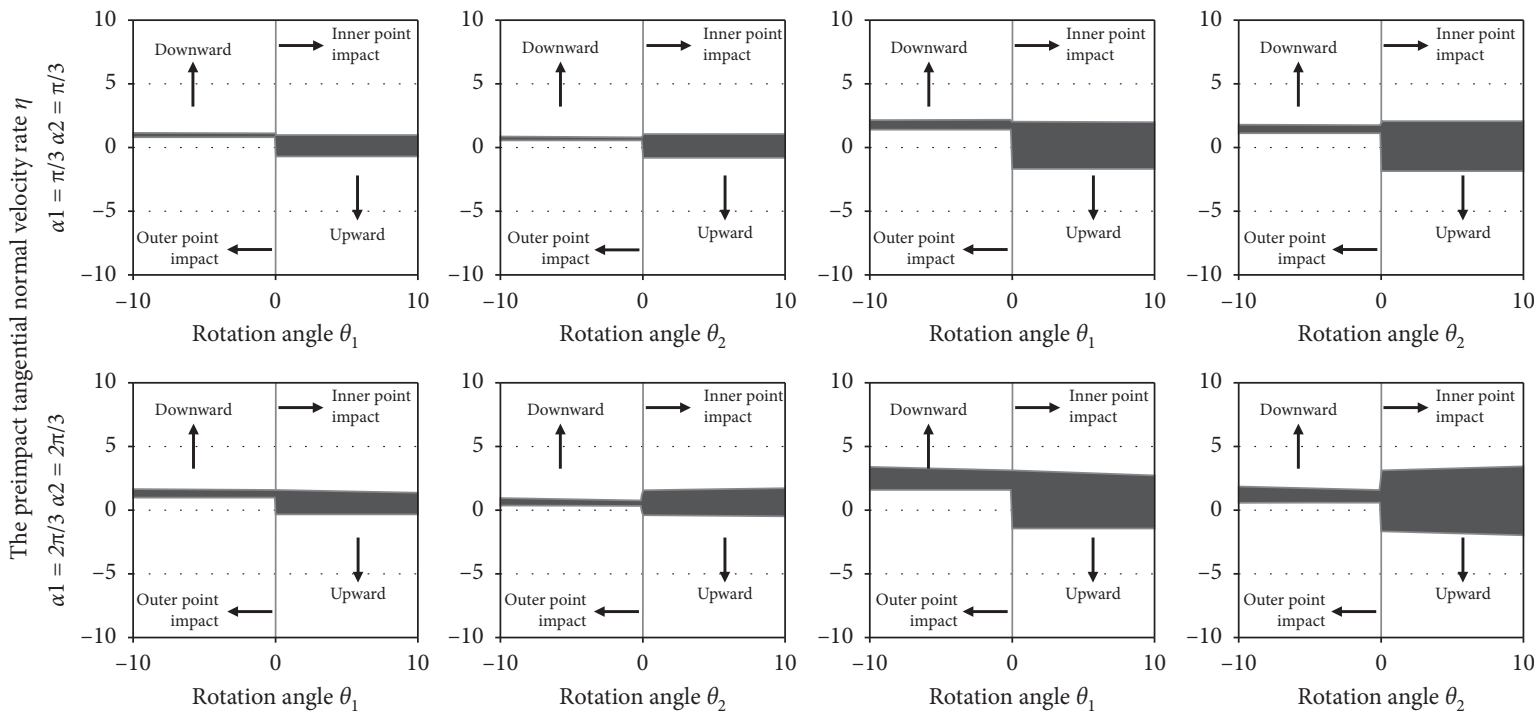

FIGURE 11: Identification of stick and upward and downward slip states for different preimpact transverse and normal velocity rates for various preimpact rotations of the center angle of girder at $60^{\circ}$ and $60^{\circ}$ (upper part) and $120^{\circ}$ and $120^{\circ}$ (lower part).

\section{Conclusions}

In this paper, the nonsmooth rigid body dynamics method and set-valued formulation are used to model all possible postimpact states of a multisegment curved bridge by using the linear complimentary formula. The single-point and multipoint impacts are described, and the preconditions and corresponding closed-form solutions for all postimpact cases are calculated.

It is concluded that the geometric characteristics and inertial conditions of the bridge segments define the location of the contact points. The preimpact tangential normal velocity rate, $\eta$, the normal impact recovery coefficient, $\varepsilon_{N}$, and the transverse friction coefficient, $\mu$, determine the state of motion between the decks after impact. This paper also proposes equations for solving the contact kinematics between two adjacent curved planar rigid bodies in all possible situations. From the conducted analyses, it is found that the impact between the segments of a curved bridge is the major factor of segment rotation.

\section{Nomenclature}

$\mu$ :

$\varepsilon_{N}$ :

LCP:

$\dot{\mathbf{g}}_{N}^{+}, \dot{\mathbf{g}}_{N}^{-}$:

$\dot{\mathbf{g}}_{T}^{+}, \dot{\mathbf{g}}_{T}^{-}$:

$v_{T R}, v_{T L}:$

$\Lambda_{N}, \Lambda_{T}:$

$\Lambda_{T R}, \Lambda_{T L}:$
Coefficient of friction

Coefficient of restitution

Linear complementary problem

Relative normal post- and preimpact velocities

Relative transverse post- and preimpact velocities

Right and left velocity parts

(Figure 2) of postimpact tangential Impulse in normal and tangential directions

Right and left impulse parts of tangential impulse that satisfy

$\Lambda_{T L}=\overline{\bar{\mu}} \Lambda_{N}-\Lambda_{T}=2 \overline{\bar{\mu}} \Lambda_{N}-\Lambda_{T R}$

$\begin{array}{ll}\mathbf{v}_{N}, \mathbf{v}_{T}: & \text { Velocity jump }\left(\mathbf{v}_{T}=\mathbf{v}_{T R}-\mathbf{v}_{T L}\right) \\ \mathbf{G}_{N N}, \mathbf{G}_{N T}, \mathbf{G}_{T N}, \mathbf{G}_{T T}: & \text { Effective mass } \\ \mathbf{M}: & \text { Mass matrix } \\ \mathbf{I}: & \text { Moment of inertia } \\ m_{1}, m_{2}: & \text { Masses of decks } \\ \theta_{1}, \theta_{2}: & \text { Angle of rotation of deck-1 and deck- } \\ \widetilde{\mathbf{r}}_{N 1}, \mathbf{\mathbf { r }}_{N 2}: & 2 \\ \mathbf{W}_{N}, \mathbf{W}_{T}: & \text { Lever arm due to rotation } \\ \mathbf{r}_{\mathrm{D}}: & \text { Normal and tangential constrained } \\ \delta: & \text { Jacobian matrix } \\ \sigma_{1}, \sigma_{2}: & \text { Distance vector between deck-1 and } \\ & \text { deck-2 } \\ & \text { Gap between two decks } \\ R_{1}, R_{2}: & \text { Parameters describing location of } \\ \alpha_{1}, \alpha_{2}: & \text { contact point at contour of another } \\ W: & \text { deck } \\ d_{m 1}, d_{m 2}: & \text { Radii of curvature of two decks } \\ x_{1}, y_{1}, x_{2}, y_{2}: & \text { Curvatures of decks } \\ \mathbf{v}_{T R}, \mathbf{v}_{T L}: & \text { Width } \\ & \text { Distance from center of mass to } \\ \eta: & \text { center of bridge curvature }(i=1,2) \\ & \text { Generalized coordinates of system } \\ & \text { Relative velocity at post- and } \\ & \text { preimpact conditions in transverse } \\ & \text { direction } \\ & \text { Preimpact tangential normal velocity } \\ & \text { rate. }\end{array}$

\section{Data Availability}

No data were used to support this study.

\section{Conflicts of Interest}

The authors declare that there are no conflicts of interest regarding the publication of this paper. 


\section{Acknowledgments}

This research was supported by the Scientific Research Fund of Institute of Engineering Mechanics, China Earthquake Administration (no. 2018A02) and the National Key R\&D Program of China (no. 2018YFC1504602).

\section{References}

[1] K. Bi, H. Hao, and N. Chouw, "Required separation distance between decks and at abutments of a bridge crossing a canyon site to avoid seismic pounding," Earthquake Engineering \& Structural Dynamics, vol. 39, no. 3, pp. 303-323, 2010.

[2] M. Miari, K. K. Choong, and R. Jankowski, "Seismic pounding between bridge segments: a state-of-the-art review," Archives of Computational Methods in Engineering, pp. 1-10. In press, 2020.

[3] H.-Y. Jia, X.-L. Lan, S.-X. Zheng, L.-P. Li, and C.-Q. Liu, "Assessment on required separation length between adjacent bridge segments to avoid pounding," Soil Dynamics and Earthquake Engineering, vol. 120, pp. 398-407, 2019.

[4] S. Pourzeynali and M. Hoseinzadeh, "An investigation on the parameters influencing the pounding in highway bridges," International Journal of Engineering, vol. 30, no. 9, pp. 1298-1308, 2017.

[5] D. Williams and W. Godden, "Seismic response of long curved bridge structures: experimental model studies," Earthquake Engineering \& Structural Dynamics, vol. 7, no. 2, pp. 107-128, 1979.

[6] A. D. Panagiotou, G. Mavromanolakis, and J. Tzoulis, "Implications of spatial variation of ground motion on collapse of SR 14/I-5 southbound and overhead bridge in Northridge earthquake," Collapse, vol. 6, no. 6, pp. 514-522, 1999.

[7] M. Shinozuka, "The Hanshin-Awaji earthquake of january 17, 1995: performance of lifelines," Technical Report NCEER-950015, p. 316, US National Center for Earthquake Engineering Research (NCEER), San Francisco, CA, USA, 1995.

[8] Y. Huang, "The performance of girder bridges in Wenchuan earthquake and a new method for seismic protection," Journal of Earthquake Engineering \& Engineering Vibration, vol. 28, no. 5 , pp. 20-26, 2008.

[9] K. Liu and L. H. Wang, "Earthquake damage of curved highway bridges in 2008 Wenchuan earthquake," Advanced Materials Research, vol. 838-841, pp. 1571-1576, 2013.

[10] Z. Sun, D. Wang, X. Guo, B. Si, and Y. Huo, "Lessons learned from the damaged Huilan interchange in the 2008 Wenchuan earthquake," Journal of Bridge Engineering, vol. 17, no. 1, pp. 15-24, 2012.

[11] M. Amjadian and A. K. Agrawal, "Rigid-body motion of horizontally curved bridges subjected to earthquake-induced pounding," Journal of Bridge Engineering, vol. 21, no. 12, Article ID 04016090, 2016.

[12] A. Banerjee, A. Chanda, and R. Das, "Seismic analysis of a curved bridge considering deck-abutment pounding interaction: an analytical investigation on the post-impact response," Earthquake Engineering \& Structural Dynamics, vol. 46, no. 2, pp. 267-290, 2017.

[13] F. D. R. Julian, T. Hayashikawa, and T. Obata, "Seismic performance of isolated curved steel viaducts equipped with deck unseating prevention cable restrainers," Journal of Constructional Steel Research, vol. 63, no. 2, pp. 237-253, 2007.

[14] J. D. Wieser, E. Maragakis, I. G. Buckle, and A. E. Zaghi, “A methodology for the experimental evaluation of seismic pounding at seat-type abutments of horizontally curved bridge," in Proceedings of the 15th World Conference on Earthquake Engineering, Chicago, IL, USA, March 2012.

[15] M. Miari, K. K. Choong, and R. Jankowski, "Seismic pounding between adjacent buildings: identification of parameters, soil interaction issues and mitigation measures," Soil Dynamics and Earthquake Engineering, vol. 121, pp. 135-150, 2019.

[16] A. Banerjee, A. Chanda, and R. Das, "Historical origin and recent development on normal directional impact models for rigid body contact simulation: a critical review," Archives of Computational Methods in Engineering, vol. 24, no. 2, pp. 397-422, 2017.

[17] S. A. Anagnostopoulos, "Pounding of buildings in series during earthquakes," Earthquake Engineering \& Structural Dynamics, vol. 16, no. 3, pp. 443-456, 1988.

[18] S. A. Anagnostopoulos and K. V. Spiliopoulos, "An investigation of earthquake induced pounding between adjacent buildings," Earthquake Engineering \& Structural Dynamics, vol. 21, no. 4, pp. 289-302, 1992.

[19] S. Muthukumar and R. DesRoches, "A Hertz contact model with non-linear damping for pounding simulation," Earthquake Engineering \& Structural Dynamics, vol. 35, no. 7, pp. 811-828, 2006.

[20] K. Ye, L. Li, and H. Zhu, "A note on the Hertz contact model with nonlinear damping for pounding simulation," Earthquake Engineering \& Structural Dynamics, vol. 38, no. 9, pp. 1135-1142, 2009.

[21] J. J. Moreau, "Unilateral contact and dry friction in finite freedom dynamics," Nonsmooth Mechanics and Applications, Springer, Vienna, pp. 1-82, 1988.

[22] P. D. Panagiotopoulos, "Nonconvex energy functions. Hemivariational inequalities and substationarity principles," Acta Mechanica, vol. 48, no. 3-4, pp. 111-130, 1983.

[23] P. D. Panagiotopoulos, "Dynamic and incremental variational inequality principles, differential inclusions and their applications to co-existent phases problems," Acta Mechanica, vol. 40, no. 1-2, pp. 85-107, 1981.

[24] É. Delassus, "Mémoire sur la théorie des liaisons finies unilatérales," Annales Scientifiques de l'École Normale Supérieure, vol. 34, pp. 95-179, 1917.

[25] F. Pfeiffer and C. Glocker, Multibody Dynamics with Unilateral Contacts, John Wiley \& Sons, Hoboken, NJ, USA, 1996.

[26] E. G. Dimitrakopoulos, "Analysis of a frictional oblique impact observed in skew bridges," Nonlinear Dynamics, vol. 60, no. 4, pp. 575-595, 2010.

[27] E. G. Dimitrakopoulos, "Nonsmooth analysis of the impact between successive skew bridge-segments," Nonlinear Dynamics, vol. 74, no. 4, pp. 911-928, 2013.

[28] C. E. Lemke, "The dual method of solving the linear programming problem," Naval Research Logistics Quarterly, vol. 1 , no. 1 , pp. $36-47,1954$.

[29] R. W. Cottle and G. B. Dantzig, "Complementary pivot theory of mathematical programming," Linear Algebra and its Applications, vol. 1, no. 1, 1967.

[30] A. Banerjee, A. Chanda, and R. Das, "Oblique frictional unilateral contacts perceived in curved bridges," Nonlinear Dynamics, vol. 85, no. 4, pp. 2207-2231, 2016. 